\title{
Cavitation Damage in Flowing Liquid Sodium Using Venturi Test Section*
}

\author{
Teddy ARDIANSYAH**, Minoru TAKAHASHI***, Makoto ASABA**** \\ and Kuniaki MIURA**** \\ ** Department of Nuclear Engineering, Tokyo Institute of Technology \\ 2-12-1 N1-18, O-okayama, Meguro-ku, Tokyo, 152-8550, Japan \\ E-mail: ardiansyah.t.aa@m.titech.ac.jp \\ ${ }^{* * *}$ Research Laboratory for Nuclear Reactors, Tokyo Institute of Technology \\ 2-12-1 N1-18, O-okayama, Meguro-ku, Tokyo, 152-8550, Japan \\ **** Sukegawa Electric Co., Ltd. \\ 3-19-5, Namekawahontyo, Hitachi, Ibaraki, 317-0051, Japan
}

\begin{abstract}
The present paper describes the damage behavior caused by cavitation in flowing liquid sodium using venturi test section. The venturi test section was made from $316 \mathrm{SS}$ with an inner diameter of $6.5 \mathrm{~mm}$ and a length of $20 \mathrm{~mm}$. The experiment was conducted with pressure of argon cover gas in the expansion tank ranging from $105-110 \mathrm{kPa}$. The liquid sodium temperature was kept at $200^{\circ} \mathrm{C}$ and the sodium flow rate was $27-28 \mathrm{~L} / \mathrm{min}$, which corresponded to developed cavitation condition with the cavitation coefficient of $0.59-0.51$. This condition was kept for 600 hours during the experiment. The results showed that cavitation bubbles collapse created damage on the inner surface of the venturi test section in the form of pits due to eroded surfaces. Under SEM, most of the pits on the inner surface of the venturi test section were in the sizes of around $25 \mu \mathrm{m}$. Some pits with sizes larger than 25 $\mu \mathrm{m}$ were also observed with the largest diameter around $110 \mu \mathrm{m}$ which indicated intense collapse of cavitation bubbles. Observation on the inner surface of the test section also indicated some micro cracks, which might be caused by the cyclic process of the formation and collapse of the bubbles during cavitation process.
\end{abstract}

Key words: Sodium, Cavitation, Venturi, 316 SS, Erosion

\section{Introduction}

Erosion caused by cavitation in liquid sodium is one of the critical problems for developing Sodium-Cooled Fast Reactor (SFR) ${ }^{(1)}$. Damage of structural material caused by the collapse of cavitation bubbles in SFR may be expected in cases where the design requires a more compact reactor vessel and components, leading to higher sodium flow velocity. The cavitation might occur in local condition where the local pressure falls below the vapor pressure of the fluid due to high flow velocity. The damage of the structural material such as cracks or severe erosion leads to the failure of the reactor component. If the damage occurs in primary coolant pipes, the leak of reactor coolant from primary circuits could take place.

Previous researches have been conducted to study the damage of liquid metal cavitation, as a result, it has been known that cavitation causes damages on the surface of the materials ${ }^{(2-9)}$. For the study of liquid metal erosion, cavitation tests were conducted

${ }^{*}$ Received 25 Aug., 2010 (No. 10-0376) [DOI: 10.1299/jpes.5.77]

Copyright $\odot 2011$ by JSME 
mostly by using vibratory apparatus since it can be done at relatively low cost, the liquid metals inventory can be easily operated and handled, and a large number of data can be obtained for various combinations of liquid metals and test materials for relatively short duration of time.

However, cavitation tests using the vibratory apparatus lack the information of the actual damage process in practical flowing condition, since most of the components that suffer from cavitation are operated in the flowing systems. The formation sites and collapse sites of cavitation bubbles in flowing systems are different from each other compared to vibratory apparatus where the formation and collapse sites are approximately the same. Moreover, it is known that pitting damages formed by cavitation are influenced by the flow of the fluid ${ }^{(10)}$, and most of the data for cavitation damage in liquid sodium are obtained for short period of time and by using vibratory apparatus. Therefore it is necessary to know in more detail the damage on the surface of the test section caused by cavitation in flowing liquid sodium.

Therefore, in the present study, the damage behavior was investigated for flowing liquid sodium in a venturi test section made from 316 SS for long time period of 600 hours. The inner surfaces of the test section are observed using an optical micrograph and a scanning electron microscope (SEM) to investigate the erosion process.

\section{Nomenclature}

$K \quad$ : Cavitation coefficient

$P_{0} \quad$ : Static pressure in downstream of venturi

$P_{1}:$ Static pressure in venturi

$P_{v}:$ Saturation vapor pressure

$T$ : Liquid sodium temperature

$V_{0}$ : Liquid sodium velocity in downstream of venturi

$V_{1} \quad$ : Liquid sodium velocity in venturi

\section{Greek symbol}

$\rho \quad$ : Liquid sodium density at a given temperature

\section{Experiment}

\subsection{Experimental apparatus}

The experiment was conducted using a sodium loop facility at Sukegawa Electric Co., Ltd. (Fig. 1). Argon gas was used as a cover gas pressure in the loop during the experiment. The static downstream pressure was measured by using a pressure transducer and the flow rate of liquid sodium was measured by using an electromagnetic flow meter. The test section for this experiment is a venturi made from $316 \mathrm{SS}$ with an inner diameter of $6.5 \mathrm{~mm}$, an outer diameter of $21 \mathrm{~mm}$, and a length of $20 \mathrm{~mm}$ (Fig. 2). The detail of this apparatus can be found in Ref. (11). The gas injector at the test section was used for other experiment (not included in this paper).

\subsection{Experimental procedure}

The experimental conditions for sodium cavitation damage experiment are listed in Table 1. The physical and thermodynamic properties of sodium and the chemical composition of $316 \mathrm{SS}$ can be seen in Tables 2 and 3, respectively. The detailed 
experimental procedures are explained as follows:

1) Liquid sodium was circulated in the sodium flow loop with a desired temperature of $200^{\circ} \mathrm{C}$.

2) The circulation was stopped shortly by turning off the EMP (Electromagnetic Pump). Then, the cover gas pressure in the expansion tank was controlled to the desired experimental condition by controlling argon gas in the expansion tank to $105-110 \mathrm{kPa}$ (stagnant pressure). The stagnant pressure was defined by the measured argon gas pressure in the expansion tank when the liquid sodium was not circulated.

3) The EMP was turned on again to circulate the liquid sodium.

4) The EMP voltage was increased gradually to increase the flow rate to $27-28 \mathrm{~L} / \mathrm{min}$ until cavitation became developed in the test section which was indicated by its distinct and high noise sounds. A developed cavitation condition was set up, where the cavitation coefficient defined by Eq. (1) had the value of $0.59-0.51$. This condition was maintained for 600 hours during the experiment.

$$
K=\frac{P_{0}-P_{v}}{\frac{\rho}{2}\left(V_{l}^{2}-V_{0}^{2}\right)}
$$

5) After the experiment, the venturi test section was cut and washed by alcohol and water to remove the remaining sodium that might adhere to the surface of the test section. Then the surface of the test section was analyzed by using an optical micrograph and SEM (Scanning Electron Microscope).

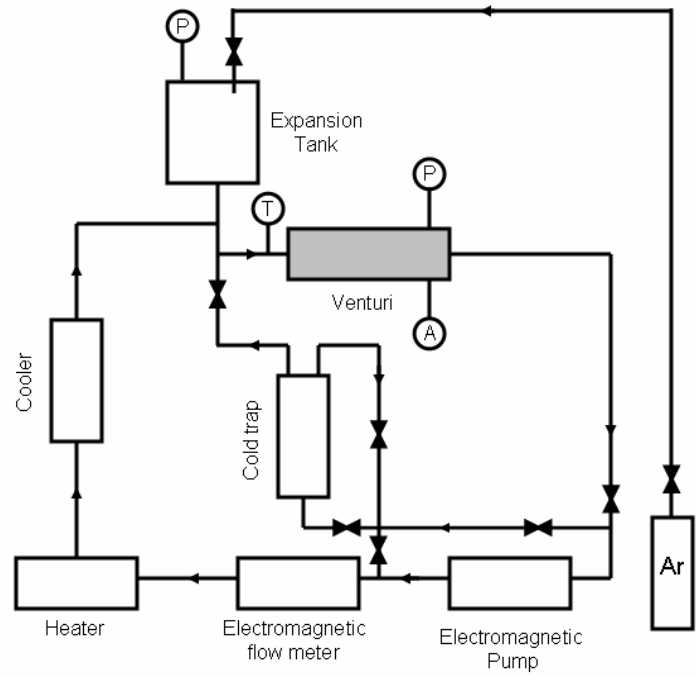

P: Pressure transducer; A: Accelerometer; T: Thermocouple

Fig. 1 Schematic of sodium loop facility.

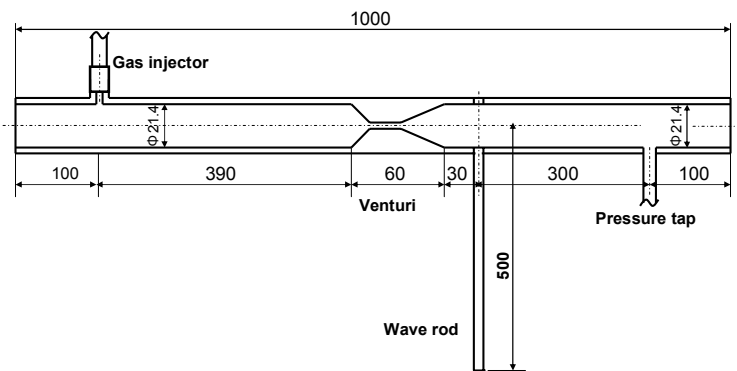

(a) Whole of test section

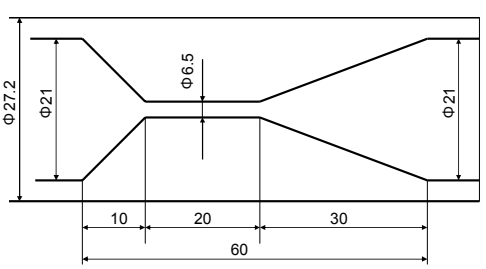

(b) Venturi

Fig. 2 Schematics of liquid sodium erosion test section. The lengths are in the unit of mm. 
Table 1. Experimental conditions for cavitation erosion experiment.

\begin{tabular}{|l|c|}
\hline Liquid sodium temperature $\left[{ }^{\circ} \mathrm{C}\right]$ & 200 \\
\hline Stagnant pressure at expansion tank $[\mathrm{kPa}]$ & $105-110$ \\
\hline Flow rate $[\mathrm{L} / \mathrm{min}]$ & $27-28$ \\
\hline Cavitation coefficient, $K[-]$ & $0.59-0.51$ \\
\hline Experimental time $[\mathrm{h}]$ & 600 \\
\hline
\end{tabular}

Table 2. Physical and thermodynamic properties of liquid sodium ${ }^{(12)}$

\begin{tabular}{|l|c|}
\hline Melting point $\left[{ }^{\circ} \mathrm{C}\right]$ & 98 \\
\hline Boiling point $\left[{ }^{\circ} \mathrm{C}\right]$ & 883 \\
\hline Vapor pressure at $200^{\circ} \mathrm{C}[\mathrm{MPa}]$ & $2.20 \times 10^{-8}$ \\
\hline Liquid density at $200^{\circ} \mathrm{C}\left[\mathrm{kg} \cdot \mathrm{m}^{-3}\right]$ & 903 \\
\hline
\end{tabular}

Table 3. Compositions of $316 \mathrm{SS}$.

\begin{tabular}{|c|c|}
\hline Material & $\%$ \\
\hline $\mathrm{Fe}$ & Balance \\
\hline $\mathrm{C}$ & $0.04-0.1$ \\
\hline $\mathrm{Mn}$ & $0.04-0.1$ \\
\hline $\mathrm{Si}$ & $0.0-0.75$ \\
\hline $\mathrm{P}$ & $0.0-0.045$ \\
\hline $\mathrm{S}$ & $0.0-0.03$ \\
\hline $\mathrm{Cr}$ & $16.0-18.0$ \\
\hline $\mathrm{Mo}$ & $2.0-3.0$ \\
\hline $\mathrm{Ni}$ & $10.0-14.0$ \\
\hline
\end{tabular}

\section{Results and Discussion}

In order to see the inner surface condition of the venturi test section before cavitation test, a hole with the same diameter of $6.5 \mathrm{~mm}$ was made in a $316 \mathrm{SS}$ rod by the same machining method as that for the fabrication of the venturi test section as shown in Fig. 3(a). The cylinder was then cut into half in the axial direction, and the inner surface of the cylinder was observed by using SEM as shown in Fig. 3(b). Under SEM observation, clear lines made by machining were observed on the inner surface, and no indication of pits or erosion can be observed.

In this experiment, the venturi test section was tested in developed cavitation condition using liquid sodium for 600 hours. After experiment, it was washed by using alcohol and water, and cut into half in the horizontal direction as shown in Fig. 3c. The cut part of the test section was polished smoothly to observe the damage part from its cross section. From visual observation using naked eyes on the inner surface of the cross section, there were not found any eroded parts appreciably. However, color degradation from white-colored surface to brown-colored one was observed on inner surface in the downstream part, which suggests the occurrence of erosion. Since cavitation bubbles are formed at the venturi part, transported downstream through the venturi part, and collapse in the downstream part due to the recovery of liquid sodium static pressure.

In order to observe the inner surface of the test section more clearly, an optical micrograph was used and the result is shown in Fig. 4. The upper picture of Fig. 4 shows the upstream part of the venturi where cavitation bubbles are formed due to the decrease in static pressure below the vapor pressure of liquid sodium at $200^{\circ} \mathrm{C}$ because of the acceleration of liquid sodium flow. This acceleration occurs when liquid sodium travels from the upstream part to the inside of the venturi region. The figure shows that the inner surface at the upstream part of the venturi reveals relatively smooth surface. No clear micro pits are observed in this region. The lower picture of Fig. 4 shows the downstream part of the venturi. The surface shows some pitting damages at around $100 \mu \mathrm{m}$ from the throat outlet. These damages are caused by the collapse of cavitation bubbles when travelling out from the venturi. The collapse produces shock waves and jets which impinge on the surface of the test section, hence creating some observable micro pits. 


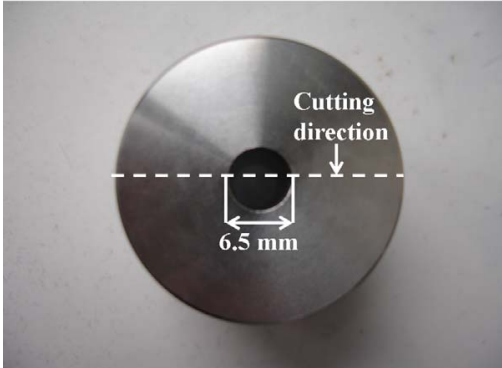

(a)

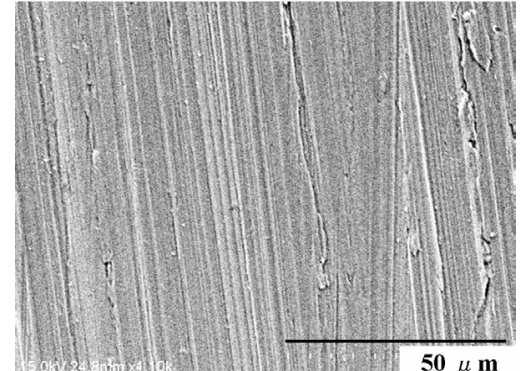

(b)

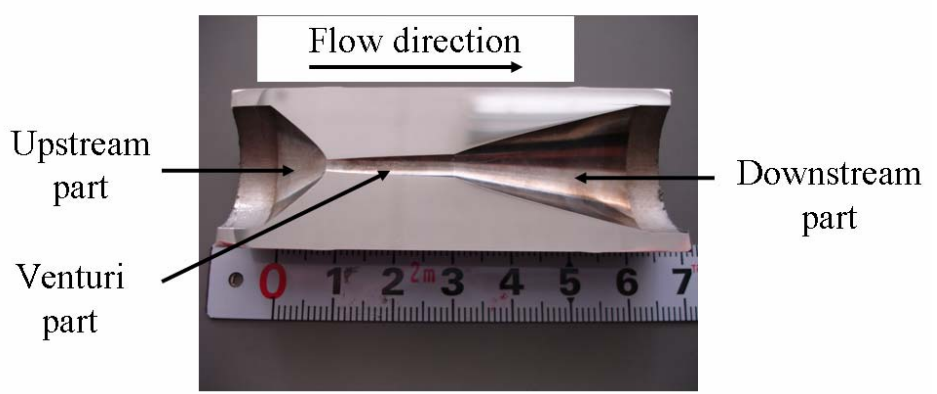

(c)

Fig. 3(a) A 316 SS cylinder made with the same machining method as the venturi test section used for cavitation damage experiment; (b) SEM of the inner surface of Fig. 3(a); and (c) Venturi test section cut into half after cavitation experiment for 600 hours.
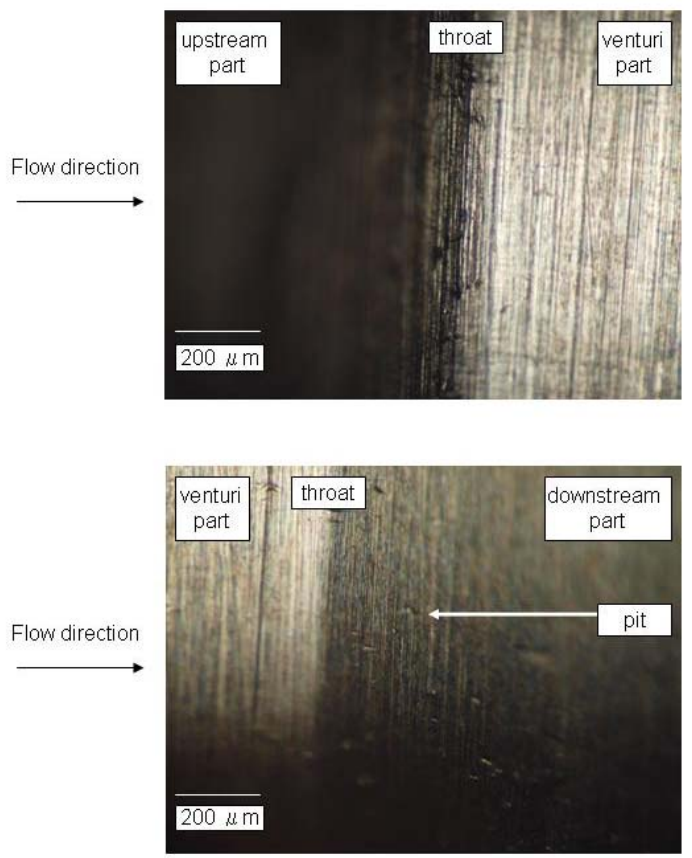

Fig. 4 Photograph of the test section surface at upstream (upper picture) and downstream (lower picture) parts of the venturi test section.

SEM was utilized to observe the damage part more clearly such as the size and shape of each pit. However, since the sample holder of the SEM machine has limited size, the test section was cut into seven samples with 5 and $10 \mathrm{~mm}$ in length (Fig. 5) so that the surface of each sample could be observed. Because of the curvature of the inner surface of the test section, observation on the surface of the test section cannot be done over the radial direction (from bottom to top). Therefore, only around the middle part of the inner surface of the test section on the axial direction was observed (from left to right). 


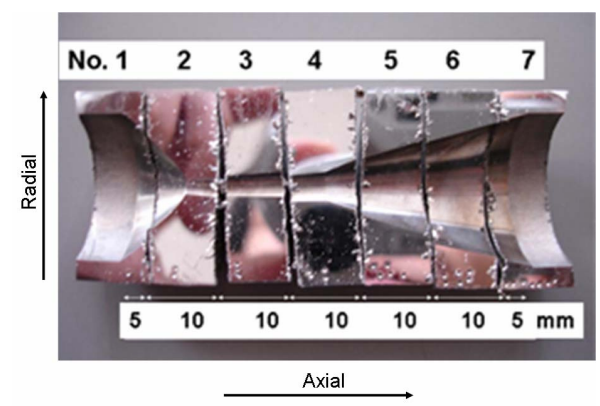

Fig. 5 Venturi test section cut into seven samples.

From the SEM observation on the surface, the number of pits on the surface of each sample was counted. Figure 6 shows the average number of pits on the surface of samples No. 4 to 7 (downstream part of the test section) where cavitation bubbles collapse due to high static pressure of the liquid sodium on these regions. It was found that the average numbers of pits were high on the surface of samples No. 6 and 7, which suggested that most of the bubbles collapsed in these regions. This was possible since the static pressure of the liquid sodium in that region was higher than at regions of samples No. 4 and 5. Cavitation bubbles would have more time to travel downstream because the pressure difference was not large enough to make the bubbles to collapse; hence the energy to produce a micro pit was not so large. Therefore, most of the bubbles might collapse at region of samples No. 6 and 7. The existence of pits on the surface of samples No. 4 and 5 suggests that some small number of bubbles also collapse there. Smaller bubbles might collapse easily on the surface of samples No. 4 and 5, while larger bubbles require larger pressure difference to collapse. Therefore, the collapse of larger bubbles can be achieved at region of samples No. 6 and 7 .

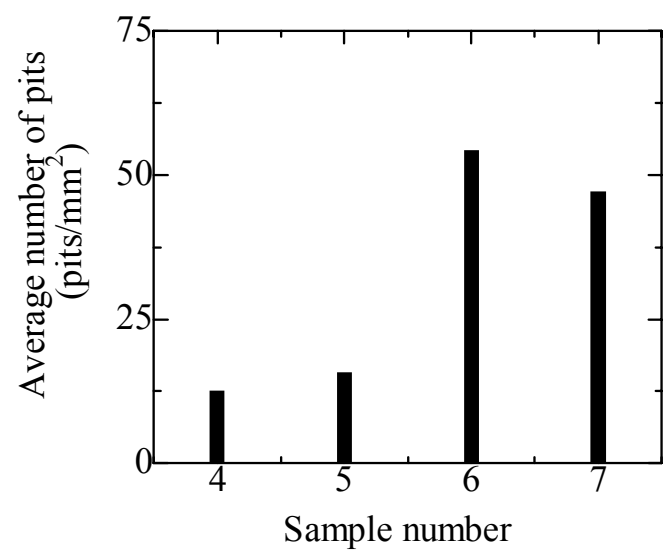

Fig. 6 Average number of pits for each sample number.

Figure 7 shows the distribution of the number of the pits with its diameter for samples No. 4 to 7. It was found that most of the pits formed on the surface of the test section had diameter of around $25 \mu \mathrm{m}$ indicated by some clearly observable peaks for samples No. 4 to 7. Samples No. 6 and 7 had larger number of pits with diameter of around $25 \mu \mathrm{m}$ compared to others. Pits with smaller diameter might be produced by the low energy from cavitation bubbles collapse, while those with larger diameter might be produced by larger energy since the collapse energy from a cavitation bubble has linear relationship with a pit being formed on the surface of the material ${ }^{(13)}$. For instance, in case of sample No. 6, some pits with diameter larger than $25 \mu \mathrm{m}$ were also observed with the largest diameter of around $110 \mu \mathrm{m}$ which indicated intense collapse of cavitation bubbles at this region which formed a larger pit diameter. 


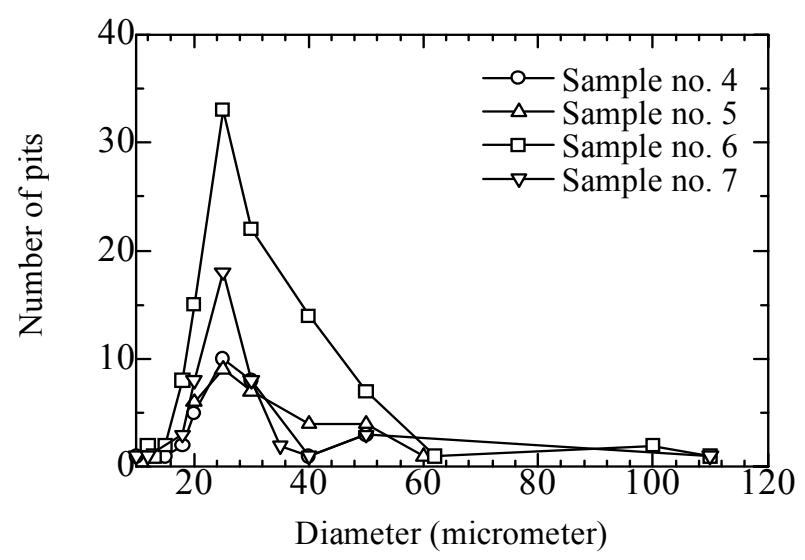

Fig. 7 Average number of pits for each sample versus its diameter.

Figure 8 shows the SEM photographs of the inner surface on sample No. 6. As shown in Fig. 8(a), pits are not distributed uniformly on the surface. Some of the pits have large diameters but others have smaller diameters. The difference in the diameter of the pits might be caused by the difference of the energy absorbed by the material to form a pit resulting from the collapse of a cavitation bubble. Since the collapse of cavitation bubbles occurred randomly, therefore it was expected statistically that a pit was formed by a single collapse of cavitation bubble ${ }^{(14)}$. However, larger pits might be produced by the collapsing of some small cavitation bubbles at the same local place or by larger bubbles although the probability was very small. Therefore it can be seen in Fig. 7 above that the number of pits with diameter larger than $25 \mu \mathrm{m}$ is decreasing.

Meanwhile, Fig. 8(b) shows an enlarged view of a pit with a diameter of around $25 \mu \mathrm{m}$. It seems that the ductile surface is deformed to a pit by pressure pulse. From the surface photograph using SEM, the depth of the pit could not be determined due to the curvature of the test section.

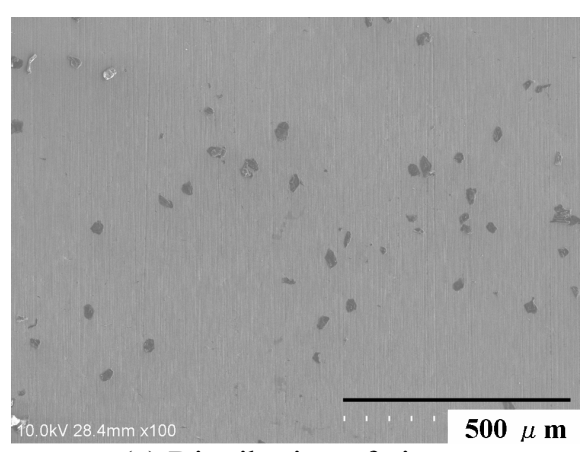

(a) Distribution of pits

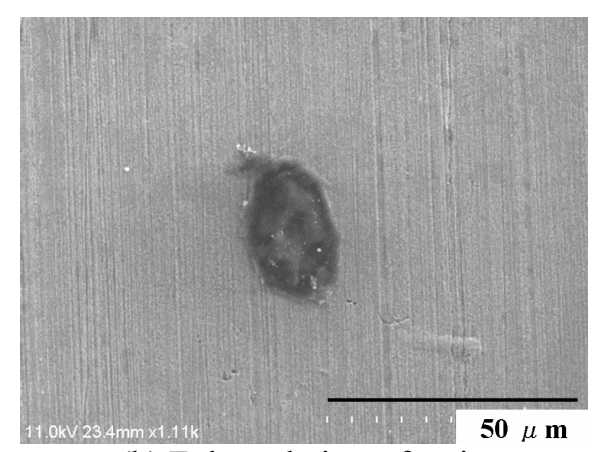

(b) Enlarged view of a pit

Fig. 8 SEM of inner surface on sample No. 6.

Besides micro pits which were formed on the inner surface of the test section, some micro cracks were also observed on the inner surface of samples No. 2, 3 and 4 with the length of around $100 \mu \mathrm{m}$ as shown in Fig. 9. These micro cracks might be caused by the cyclic process that occurred on the test section due to the formation and collapse of the bubbles at the test section. It was known that the formation and collapse of cavitation bubbles in venturi test section occurred with certain frequency ${ }^{(15)}$. When this condition was maintained for longer time scale, material became fatigue and small cracks might grow slowly into larger ones. If these cracks cannot be prevented well, material can be damaged further which could cause an accident during the operation of a liquid sodium-cooled fast reactor. And this could prevent the operation of reactor for a longer time. 


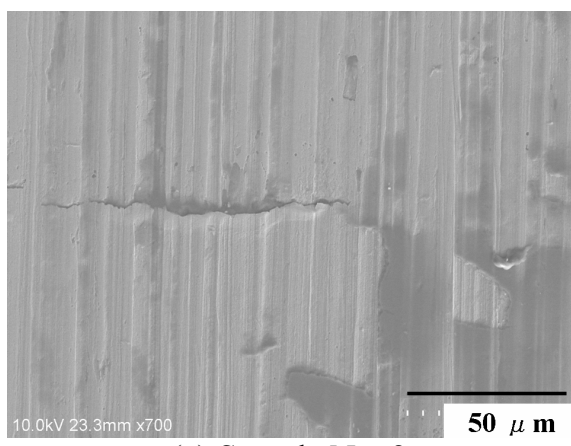

(a) Sample No. 2

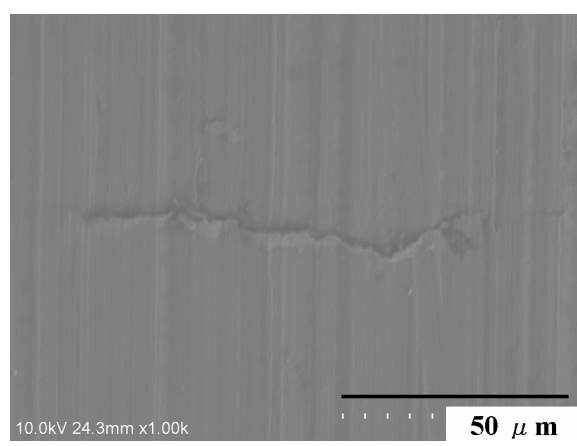

(b) Sample No. 3

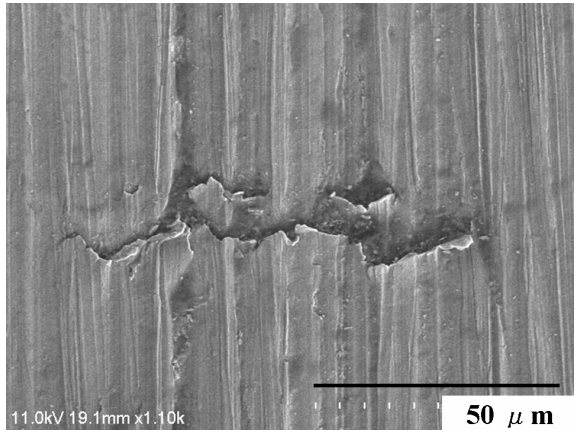

(c) Sample No. 4

Fig. 9 SEM observation of micro cracks on inner surface of the test section.

\section{Conclusion}

Cavitation erosion experiment has been conducted for flowing liquid sodium in venturi test section made from $316 \mathrm{SS}$. The following conclusions are obtained:

1) Cavitation created damage on the surface of the test section in the form of pits due to eroded surface and micro cracks.

2) Most of the pits formed on the surface of the test section occurred on sample No. 6 and 7. These pits had diameter of around $25 \mu \mathrm{m}$. Some pits with diameter larger than $25 \mu \mathrm{m}$ were also observed with the largest diameter of around $110 \mu \mathrm{m}$, which suggested intense collapse of cavitation bubbles in this region.

3) Micro cracks were observed on the inner surface of the venturi test section which might be caused by the cyclic process of the formation and collapse of the cavitation bubbles at the venturi test section.

\section{Acknowledgement}

The authors would like to express their gratitude to the Japan Nuclear Energy Safety Organization (JNES) for supporting this study financially.

\section{References}

(1) Konomura, M. and Ichimaya, M., Design Challenges for Sodium Cooled Fast Reactors, Journal of Nuclear Materials, Vol. 371 (2007), pp. 250-569.

(2) Young, S. G. and Johnston, J. R., Effect of Temperature and Pressure on Cavitation Damage in Sodium, Characterization and Determination of Erosion Resistance, ASTM STP 474, American Society of Testing and Materials (1970), pp. 67-108.

(3) Preiser, H. S., Thiruvengadam, A. and Couchman III, C. E., Cavitation Damage in Liquid Sodium, NASA CR-54071 (1964). 
(4) Thiruvengadam, A., Preiser, H. S. and Rudy, S. L., Cavitation Damage in Liquid Metals, NASA CR-54391 (1965).

(5) Thiruvengadam, A. and Preiser, H. S., Cavitation Damage in Liquid Metals, NASA CR-72035 (1965).

(6) Young, S. G. and Johnston, J. R., Effect of Cover Gas Pressures Up To 4 Atmospheres on Accelerated Cavitation Damage in Sodium, NASA TMX-61065 (1967).

(7) Young, S. G. and Johnston, J. R., Effect of Cover Gas Pressures on Accelerated Cavitation Damage, NASA TMX-52414 (1968).

(8) Smith, P. G., DeVan, J. H. and Grindell, A. G., Cavitation Damage to Centrifugal Pump Impellers during Operation with Liquid Metals and Molten Salt at 1050-1400 F. Transaction of the ASME Series D, Journal of Basic Engineering, Vol. 85 (1963), pp. 329-337.

(9) Young, S. G. and Johnston, J. R. Accelerated Cavitation Damage of Steels and Superalloys in Sodium and Mercury, Erosion by Cavitation or Impingements, ASTM STP 408, American Society of Testing and Materials (1967), pp. 186-219.

(10) Robinson, M. J. and Hammitt, F. G. Detailed Damage Characteristics in a Cavitating Venturi, Transaction of the ASME Series D, Journal of Basic Engineering, Vol. 89 (1967), pp. 161-173.

(11) Ardiansyah, T., Takahashi, M., Yoshizawa, Y., Nakagawa, M., Asaba, M. and Miura, K., The Onset of Cavitation and The Acoustic Noise Characteristics of Sodium Flow in a Venturi, submitted to Special Issue on $18^{\text {th }}$ International Conference on Nuclear Engineering, Journal of Power and Energy Systems (2010).

(12) Fink, J. K. and Leibowitz, L., Thermodynamic and Transport Properties of Sodium Liquid and Vapor, ANL/RE-95/2 (1995).

(13) Okada, T., Iwai, Y., Hattori, S. and Tanimura, N., Relation Between Impact Load and The Damage Produced by Cavitation Bubble Collapse, Wear, Vol. 184 (1995), pp. 231-239.

(14) Hammitt, F. G., Observations of Cavitation Damage in a Flowing System, Transaction of the ASME Series D, Journal of Basic Engineering, Vol. 85 (1963), pp. 347-359.

(15) Niiyama, K., Nozawa, M., Ohira, K. and Oike, M., Cavitation Instability in Subcooled Liquid Nitrogen Flows, Journal of Fluid Science and Technology, Vol. 3, No. 4 (2008), pp. 500-511. 